04

\title{
Убегающие электроны и параметрическая неустойчивость в торсатроне Ураган-3М
}

\author{
() И.К. Тарасов, М.И. Тарасов, Д.А. Ситников, В.В. Ольшанский, В.М. Листопад, Н.В. Лымарь, М.В. Гниденко \\ Национальный научный центр „Харьковский физико-технический институт“, Институт фризики плазмы, \\ 61108 Харьков, Украина \\ e-mail: itarasov@ipp.kharkov.ua
}

Поступило в Редакцию 19 июня 2017 г.

В окончательной редакции 13 января 2018 г.

Принято к публикации 30 сентября 2019 г.

Приведен сравнительный анализ спектров электромагнитных флуктуаций в периферийной области ловушки Ураган-3М, а также спектров флуктуационных колебаний плотности плазмы в зоне удержания плазмы в сравнении со спектрами модуляции потока убегающих электронов. Исследована зависимость формы спектра флуктуаций от интенсивности потока убегающих электронов. Обсуждены условия удержания и нагрева плазмы в торсатроне Ураган-3М.

Ключевые слова: убегающие электроны, параметрическая неустойчивость, электромагнитные флуктуации, нелинейное взаимодействие.

DOI: 10.21883/JTF.2020.03.48919.2390

\section{Введение}

Исследования физических процессов, возникающих в плазме под воздействием внешних электромагнитных волн, проводятся достаточно долго. Особенность этих исследований обусловлена тем, что динамика плазмы во многом определяется действием самосогласованных электромагнитных полей. С учетом этого проблема взаимодействия электромагнитных волн с плазмой является одной из фундаментальных. Актуальность подобных исследований обусловлена также рядом важных практических приложений физики плазмы: создание и нагрев плазмы высокочастотными полями и исследование устойчивости плазмы в лабораторных установках, в технологических плазменных установках и в ловушках плазмы для получения управляемых термоядерных реакций.

При малых значениях полей внешней электромагнитной волны процессы преобразования этой волны в плазменные волны можно описывать на основе линейной электродинамики. В сильных полях волны накачки такие процессы становятся нелинейными, что позволяет говорить о параметрическом воздействии электромагнитного излучения на плазму. При этом волна накачки в результате процессов индуцированного или комбинированного рассеяния на частицах плазмы трансформируется в плазменные волны, происходит нарастание внутреннего поля плазменных волн и развивается параметрическая неустойчивость.

При достаточно высоком уровне флуктуаций плазменные волны переходят в турбулентное состояние. Такие неустойчивости называют неустойчивостями пучкового типа (неустойчивость плазмы с током). Широкий класс неустойчивостей параметрического типа, как и пучковых, обусловлен относительным движением частиц плазмы.

В этой ситуации возникает вопрос о роли потоков заряженных частиц, конкретно убегающих электронов (УЭ), которые могут существовать в области удержания термоядерных установок до возникновения в них плазмы. Примером этого может служить торсатрон Ураган-3М (У-3М), в области удержания которого только при наличии магнитных полей без введения ВЧ-мощности и соответственно без плазмы были обнаружены потоки УЭ.

Присутствие в объеме удержания тороидальной магнитной ловушки потока УЭ оказывает существенное влияние на устойчивость плазменного шнура, а также зачастую вносит существенный вклад в энергетический баланс системы. В данном контексте большое значение имеет численность ускоренных частиц в плазме, которая во многом определяется величиной напряженности внешнего ускоряющего электрического поля $E$, а точнее его отношением к критическому значению $\left(E_{\text {crit }}=(\Lambda|e|) /\left(2 r_{\mathrm{D}}^{2}\right)\right.$, где $\Lambda=12-$ кулоновский логарифм, $e-$ заряд частицы, $r_{\mathrm{D}}$ - дебаевский радиус).

В нашем случае формирование потока ускоренных электронов обусловлено электрическим полем, возникающим вследствие изменения во времени напряженности удерживающего магнитного поля, при подаче импульса магнитного поля в торсатроне У-3М. При этом поток ускоряемых частиц на начальной стадии создает в объеме удержания плазму с плотностью $n_{e} \sim 10^{10} \mathrm{~cm}^{-3}$. Далее из образовавшейся плазмы частицы ускоряются в течение всего фронта импульса магнитного поля.

В работах, опубликованных ранее, приводились результаты экспериментального и теоретического исследований динамики флуктуаций, возбуждаемых в пери- 

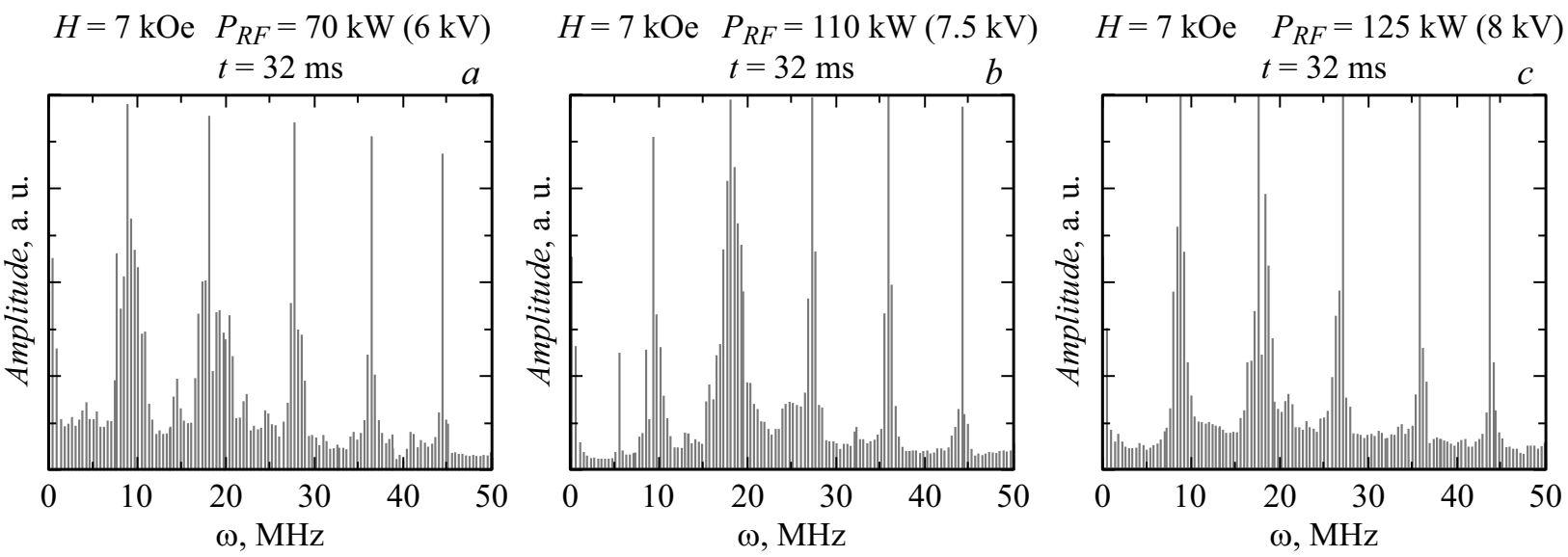

Рис. 1. Зависимость спектров флуктуаций в периферийной области торсатрона Ураган-3М от величины вводимой ВЧ мощности при напряженности магнитного поля $H=7 \mathrm{kOe}$ и временной выборке $\Delta t=32-42 \mathrm{~ms}$ от начала ВЧ импульса (60 ms), напряжении на антенне генератора „Каскад-2“. $U, \mathrm{kV}: a-6, b-7.5, c-8$.
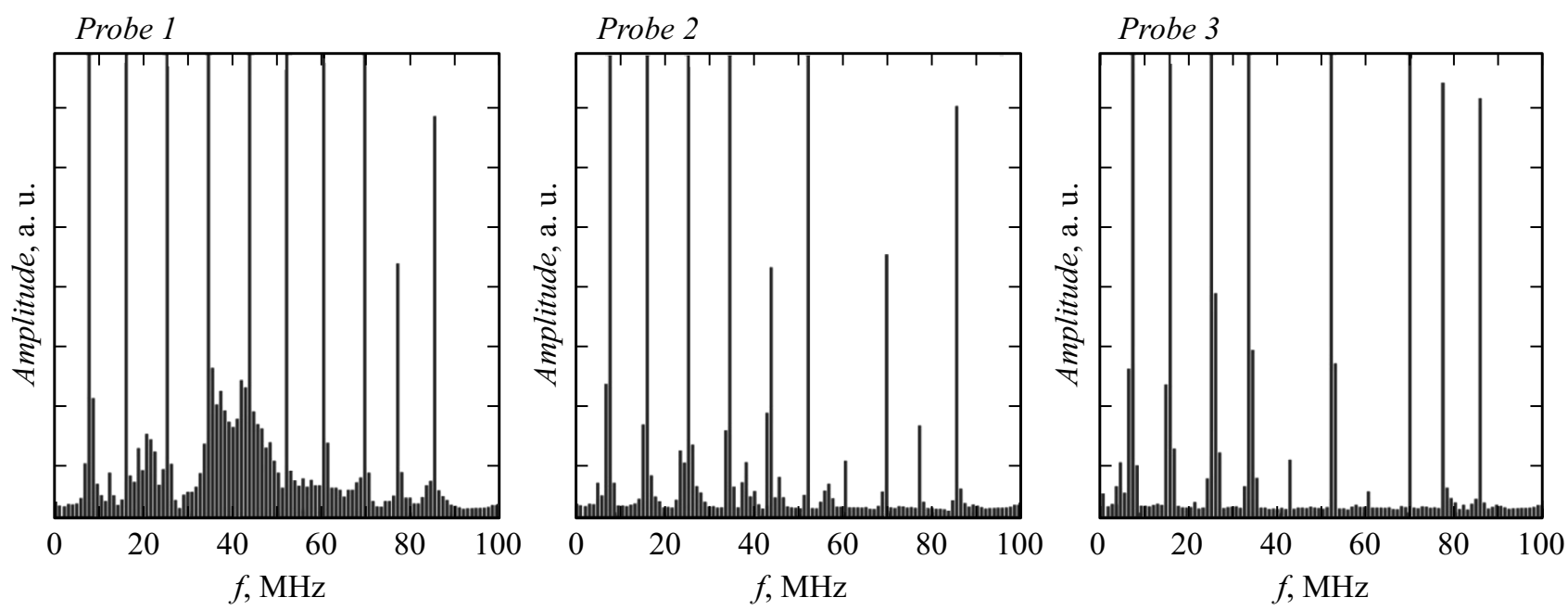

Pис. 2. Спектры сигналов, принимаемых тремя электростатическими зондами, расположенными на периферии плазменного шнура. Величина напряженности магнитного поля $(7 \mathrm{kOe})$, напряжение на антенне генератора „Каскад- $2^{“} 10 \mathrm{kV}$.

ферийной плазме малой термоядерной установки торсатрона У-3М, а также исследования причин возникновения потоков УЭ [1-3]. В работе [4] исследовалось влияние пучка электронов в плазме на величину порога возбуждения параметрической неустойчивости. Отмечено понижение порога возбуждения параметрической неустойчивости при наличии пучка электронов.

Вопрос возбуждения потоков заряженных частиц с помощью параметрической неустойчивости (бернштейновских мод) неоднократно обсуждался в научной литературе и подвергался экспериментальной проверке $[5,6]$.

В настоящей работе мы проводим сравнительный анализ спектров электростатических и магнитных флуктуаций, существующих в периферийной области объема удержания плазмы торсатрона У-3М во время создания и удержания плазмы. Также исследуются флуктуации плотности плазмы в объеме удержания плазмы. Проводится сопоставление условий возбуждения параметри- ческой неустойчивости с интенсивностью потока убегающих электронов в области удержания плазмы. Обсуждаются экспериментальные результаты, говорящие о сильном нелинейном взаимодействии потока убегающих электронов с удерживаемой плазмой и высокочастотными полями, создающими плазму в объеме удержания торсатрона.

\section{Экспериментальная установка}

Эксперименты проводились на малой термоядерной установке - торсатроне У-3М $[1,3]$. Торсатрон У-3М имеет большой радиус $R=100 \mathrm{~cm}$, удерживающее магнитное поле $B_{0} \leq 1 \mathrm{~T}$, малый радиус винтовой обмотки $r=27 \mathrm{~cm}$, заходность винтовой обмотки $l=3$, число периодов поля $m=9$, угол вращательного преобразования на крайней не разрушенной магнитной поверхности 

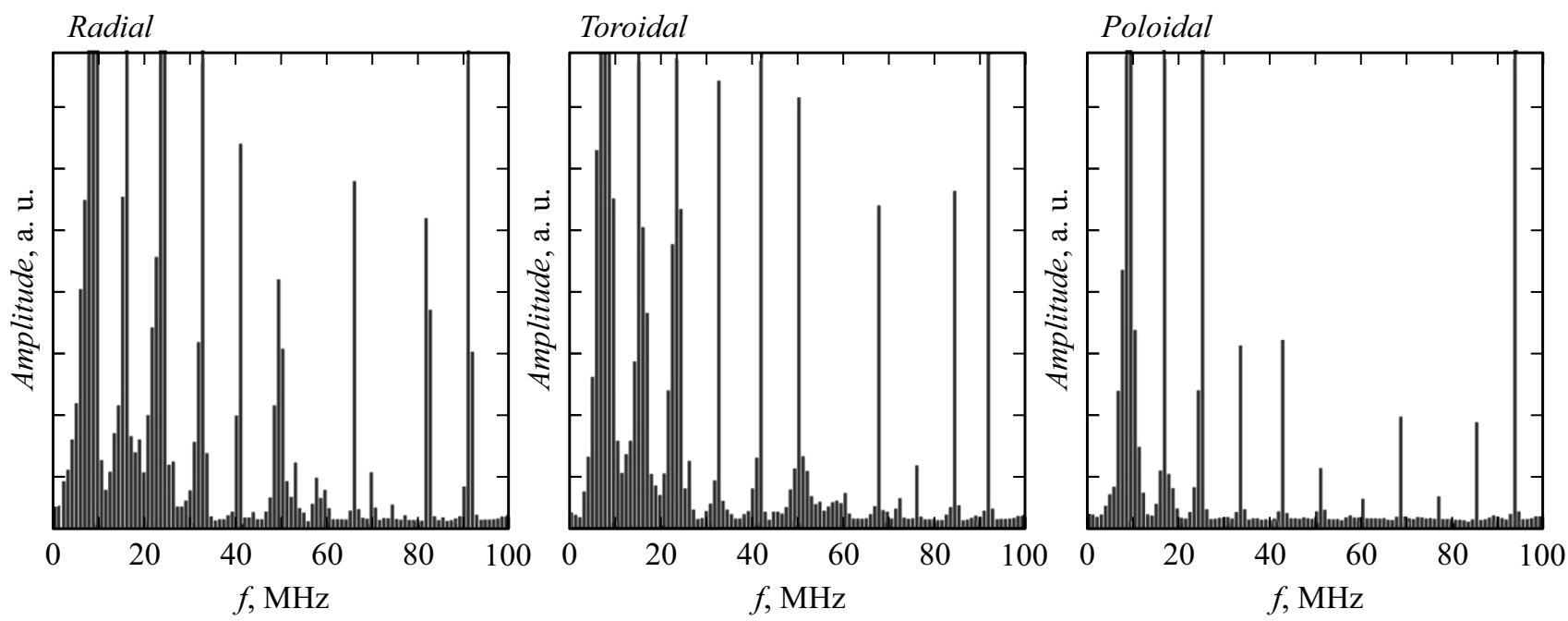

Рис. 3. Спектры сигналов с высокочастотных магнитных трехкомпонентных зондов, расположенных в периферийной области объема удержания плазмы (10 MHz/div). Напряжение на антенне генератора „Каскад-2“ $10 \mathrm{kV}$.
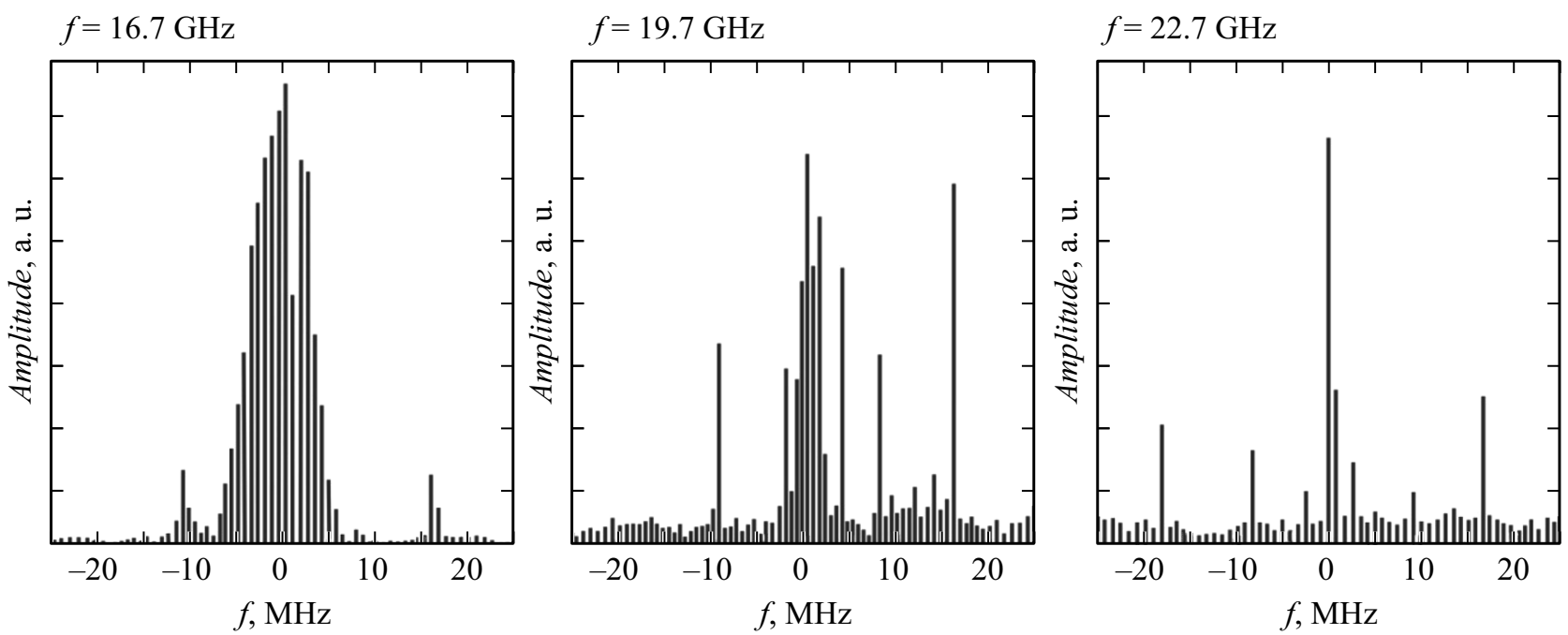

Рис. 4. Эволюция частотного спектра электромагнитной волны, отраженной от плазмы при различных частотах (5 MHz/div). Напряжение на антенне генератора „Каскад-2“ $10 \mathrm{kV}$.

$t=0.4$, угол вращательного преобразования на оси $\iota_{0}=0.18$.

Питание обмоток удерживающего магнитного поля осуществлялось с помощью генератора постоянного тока. Ток подавался на обмотки в импульсном режиме. Длительность импульса магнитного поля составляла $\sim 4$ s. При этом длительность стадии стационарного магнитного поля $2 \mathrm{~s}$, а фронты импульса магнитного поля в камере У-3М длились порядка $1 \mathrm{~s}$.

Создание и нагрев плазмы в торсатроне осуществлялся ВЧ методом, при этом ВЧ мощность вводилась в объем удержания на альфвеновской частоте $\omega=0.8^{*} \omega_{c i}$. ВЧ комплекс торсаторона У-3М предназначен для генерирования мощных высокочастотных колебаний в импульсном режиме. Он представляет собой два однотипных модуля „Каскад-1“ и „Каскад-2“, каждый из которых является генератором высокочастотных колебаний с выходной мощностью до $2 \mathrm{MW}$ в импульсе длительностью до $100 \mathrm{~ms}$. Рабочий диапазон частот генераторов $f=1-20 \mathrm{MHz}$.

Непосредственно ввод ВЧ мощности в объем удержания осуществлялся с помощью двух антенн без экранов, расположенных на противоположных секторах тора со стороны слабого магнитного поля. Для создания плазмы использовалась рамочная антенна, а для нагрева плазмы - антенна в виде трех полувитков.

В данных экспериментах магнитное поле имело величину $B=0.72 \mathrm{~T}$. Плазма в торсатроне У-3М создается и нагревается поглощением ВЧ мощности на частоте $f=8.6 \mathrm{MHz}$. Водимая мощность порядка $P \leq 200 \mathrm{~kW}$.

Для изучения электростатических флуктуаций использовался комплекс зондовой диагностики, состоя- 

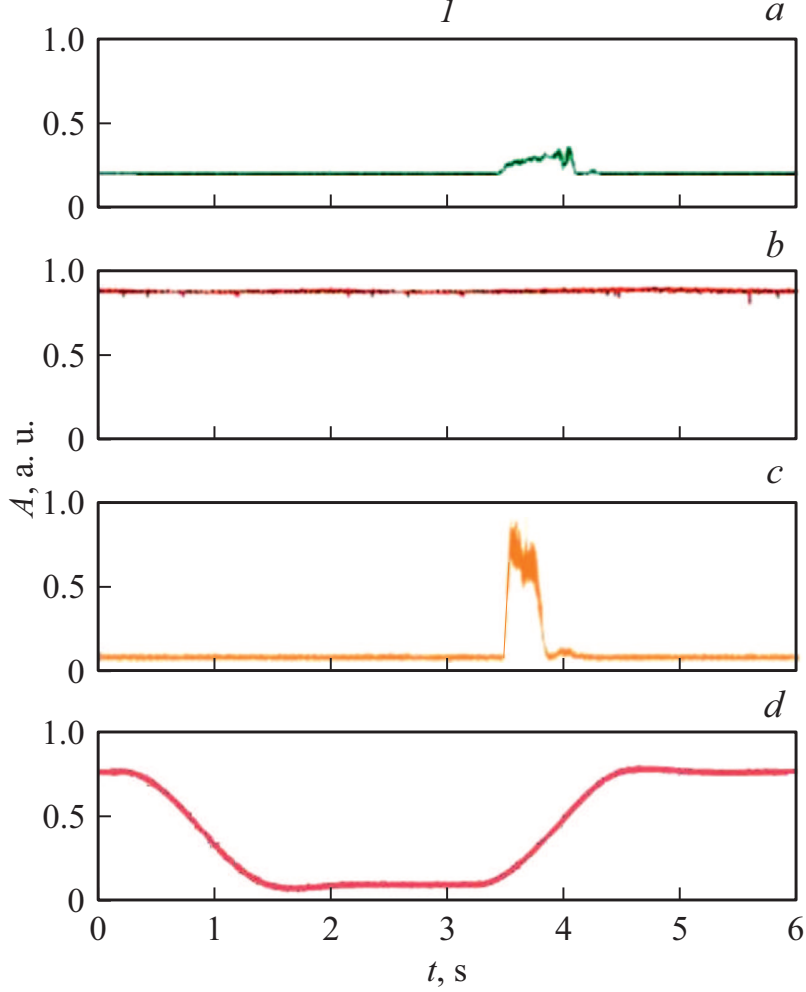
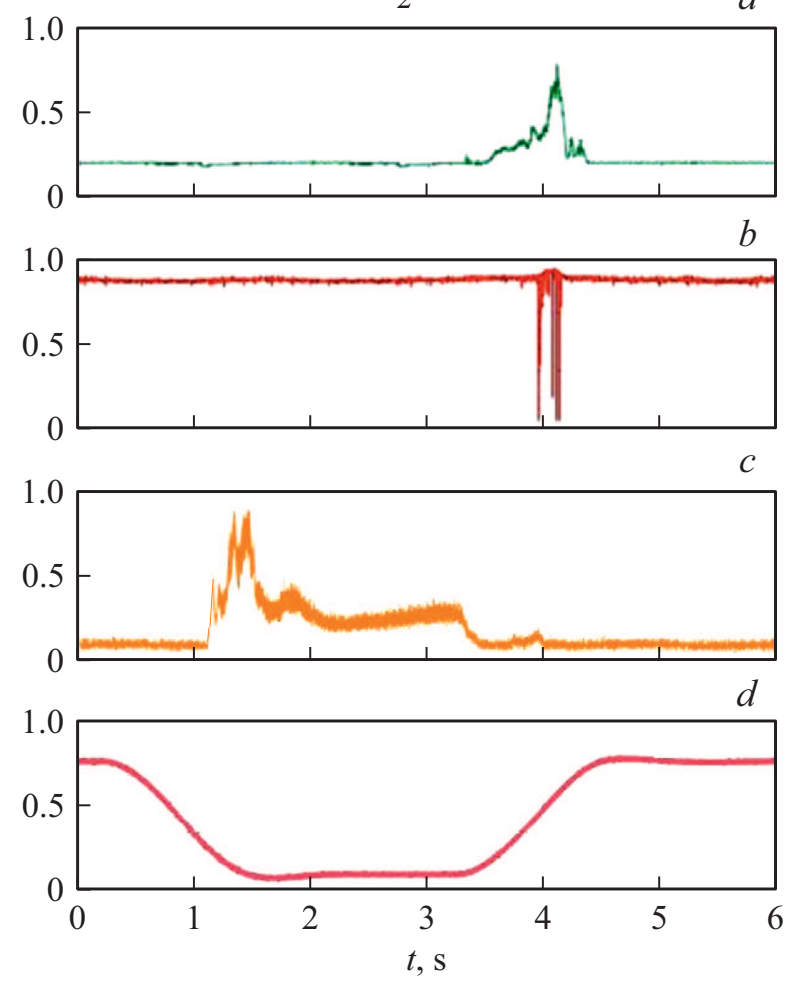

Рис. 5. Сигналы с зонда Ленгмюра (a), датчика жесткого рентгеновского излучения $(b)$, СВЧ излучения $(60 \mathrm{GHz})(c)$ и магнитного зонда $(d)$, без- и со стимулированием потока убегающих электронов $\left(1\right.$ и 2 соответственно) $\left(P=8 \cdot 10^{-5}\right.$ Torr $)$.

щий из размещенных в периферийной области неподвижных зондов с развитой поверхностью. Эти зонды, изготовленные из нержавеющей стали, имели размеры $5 \times 5 \times 0.2 \mathrm{~cm}$. Они были расположены симметрично относительно крайней замкнутой магнитной поверхности в одном из сечений плазменного шнура. Зонд 1 располагается на внутренней стороне тора в области сильного магнитного поля. Зонды 2 и 3 расположены с внешней стороны тора через $120^{\circ}$ по плоскости сечения в области слабого магнитного поля.

В том же сечении со стороны слабого магнитного поля размещены подвижные (в горизонтальном направлении) высокочастотные трехкомпонентные магнитные зонды, которые использовались в экспериментах по изучению магнитной составляющей исследуемых флуктуаций. При введении ВЧ мощности в объем удержания измерения подвижными зондами производились исключительно в периферийной области во избежание их повреждения при непосредственном контакте с плазмой.

Метод флуктуационной рефлектометрии, который также использовался в экспериментах, позволяет исследовать уровни и спектры частот колебаний в плазме. Изменение частоты излучения зондирующего СВЧ генератора давало возможность радиальной вариации критического слоя, от которого происходит отражение падающей волны. Отраженная волна несет информацию о флуктуациях плотности плазмы в области критического слоя. Этот метод хорошо зарекомендовал себя в исследованиях на токамаках. Исследование флуктуаций плотности плазмы проводились с помощью спектрального анализа модулированного СВЧ сигнала, отраженного от слоев плазмы с критической плотностью $[7,8]$.

Нами использовался вариант флуктуационной рефлектометрии с волноводным трактом, общим для падающей и отраженной волны. Сигналы по входу и выходу с волноводного тракта разделялись с помощью двойного волноводного тройника. Антенна рефлектометра располагается в сечении через $180^{\circ}$ по тору от сечения расположения магнитных и электростатических зондов, со стороны слабого магнитного поля. Сигналы со всех датчиков передавались по коаксиальным и волноводным каналам на вход анализаторов спектра (C4-59 и C4-60), которые были модернизированы для работы в импульсном режиме. Модернизация представляла собой введение в схему анализатора внешнего запуска для синхронизации с исследуемыми процессами. Использовалась возможность вывода информации с выхода „Видео“ на цифровой осциллограф.

Диагностика убегающих электронов осуществлялась по жесткому рентгеновскому излучению, возникающему в результате попадания высокоэнергичных электронов на конструктивные элементы установки (корпуса винтовой и компенсирующей обмоток, элементы крепления, вакуумная камера). Помимо рентгеновского излучения фиксировалось синхротронное излучение в широком диапазоне частот [1-3]. 


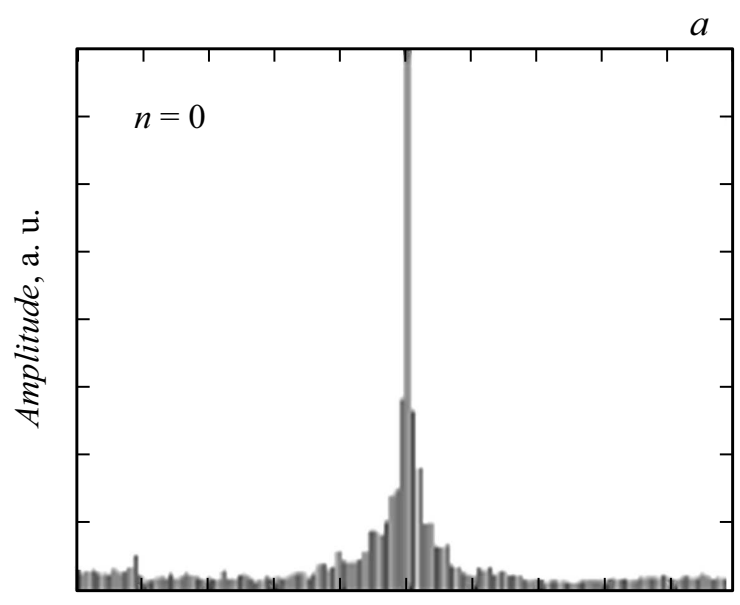

$f,(0.5 \mathrm{MHz} / \mathrm{div})$

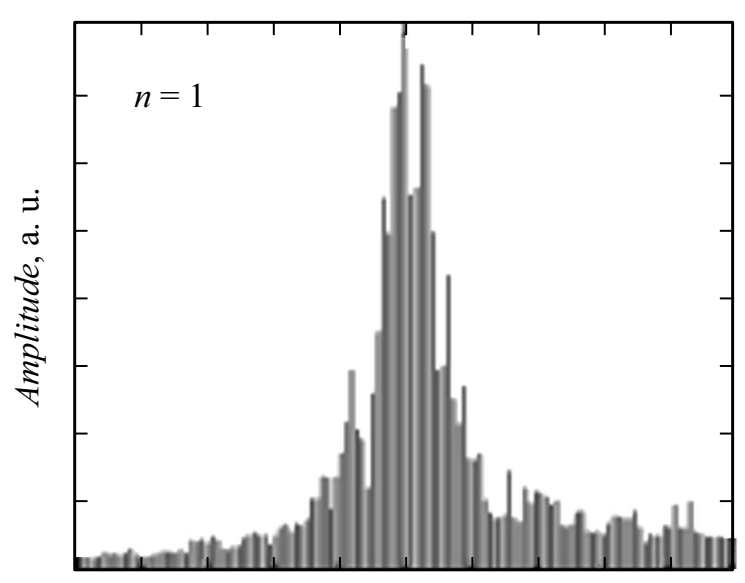

$f,(0.5 \mathrm{MHz} / \mathrm{div})$

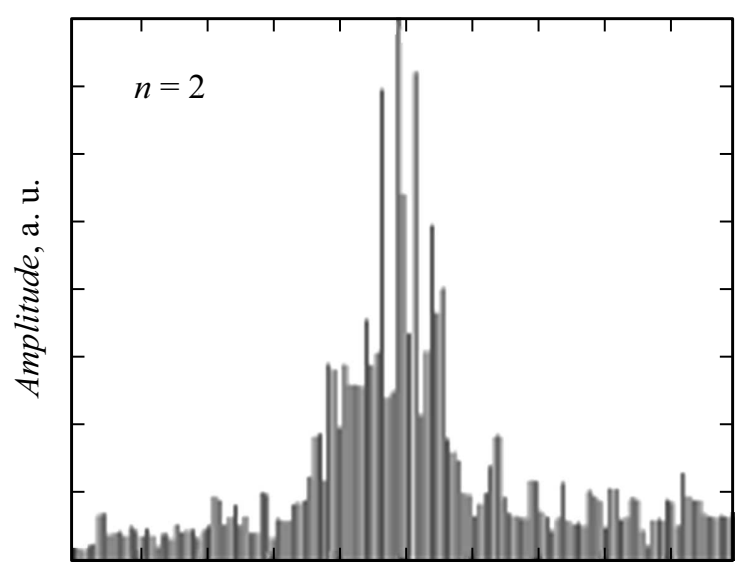

$f,(0.5 \mathrm{MHz} / \mathrm{div})$

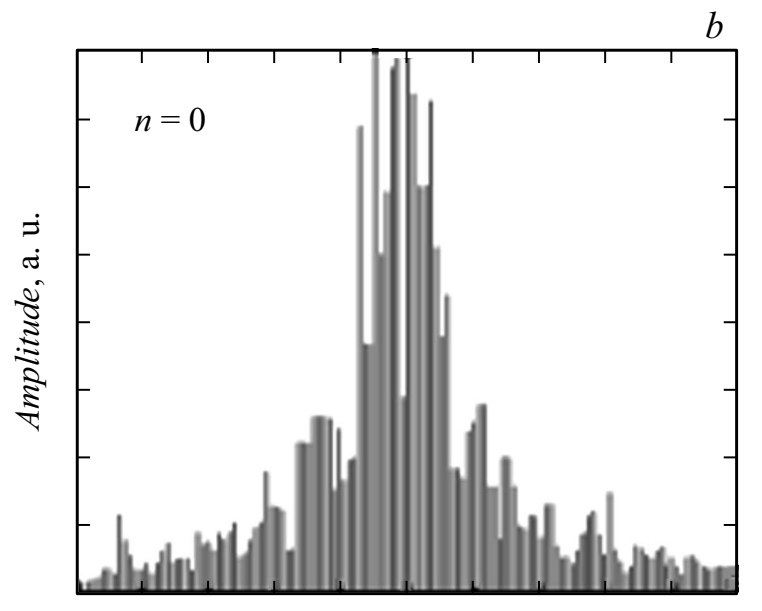

$f,(0.5 \mathrm{MHz} / \mathrm{div})$

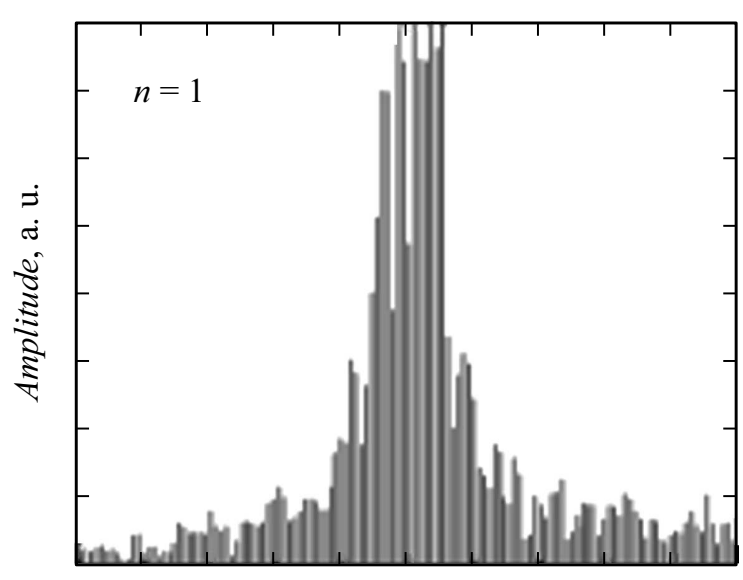

$f,(0.5 \mathrm{MHz} / \mathrm{div})$

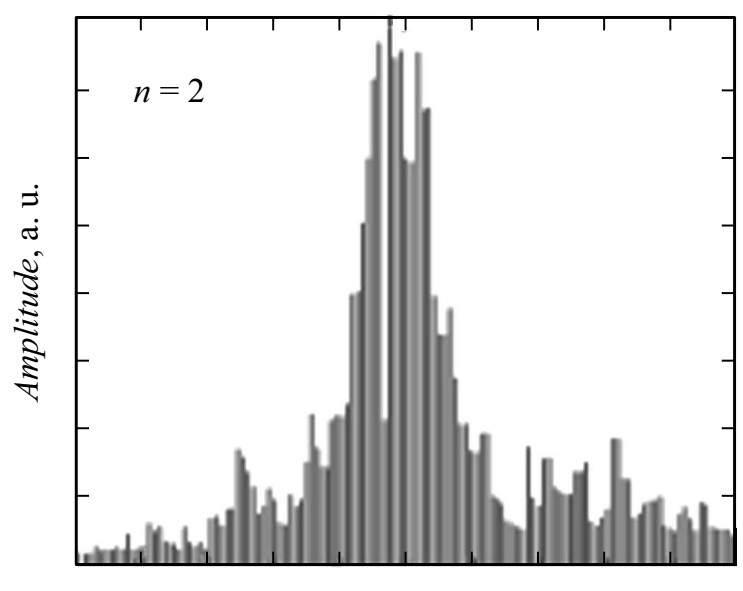

$f,(0.5 \mathrm{MHz} / \mathrm{div})$

Рис. 6. Зависимости спектров в области нулевой, первой и второй гармоник частоты ВЧ накачки от интенсивности потока убегающих электронов: $a-$ без стимулирования, $b-$ при стимулировании.

Поток УЭ на стадии их возникновения и распада диагностировался с помощью пояса Роговского, а также зондов Ленгмюра и зондов с развитой поверхностью.

Стимулирование потока УЭ производилось на переднем фронте импульса магнитного поля. В тече- ние времени существования плазмы, из которой вытягивался поток убегающих электронов, дополнительно поджигалась плазма с помощью СВЧ генератора частотой $2.45 \mathrm{GHz}$, тем самым производилась подпитка существующей плазмы, что увеличивало ее плотность, 

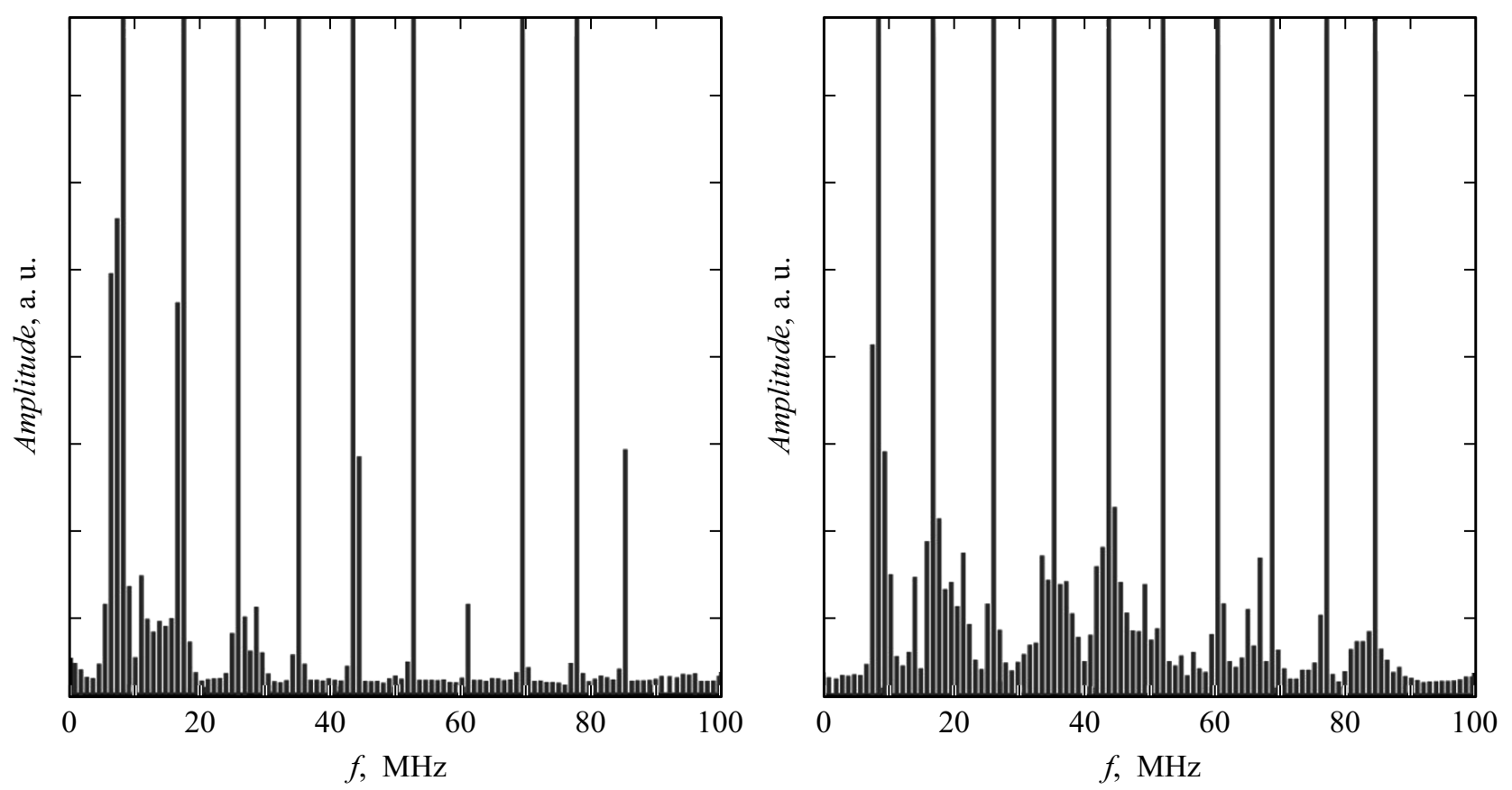

Рис. 7. Смещение спектра гармоник частоты ВЧ накачки при увеличении интенсивности потока убегающих электронов: $a-6 е з$ стимулирования убегающих электронов, $b-$ при стимулировании.

температуру и соответственно интенсивность потоков убегающих электронов.

\section{Экспериментальные результаты}

Здесь приведены результаты экспериментальных исследований колебательных процессов, наблюдаемых в периферийной области удержания плазмы. Спектры флуктуаций в граничной области удержания плазмы соответствовали гармоникам частоты высокочастотного нагрева (1-12 гармоники). Наблюдалась зависимость спектральной картины от величины вводимой высокочастотной мощности. С увеличением мощности интенсивность спектра смещалась в высокочастотную область. Это относится как к основным гармоникам, так и к частотам, не кратным основной частоте.

Необходимо также отметить, что в зависимости от задержки временной выборки $(10 \mathrm{~ms})$ от начала ВЧ импульса спектр также претерпевал изменения, что соответствовало изменению плотности плазмы в течение ВЧ импульса (рис. 1).

Спектры флуктуаций фиксировались с помощью набора из трех электростатических зондов. Из спектрограмм следует, что в одном сечении плазменного шнура спектры колебаний достаточно сильно отличаются (рис. 2).

Далее представлены спектры колебаний, зафиксированные с помощью трехкомпонентных магнитных зондов (рис. 3). Спектры радиальной и полоидальной составляющих близки по форме, но форма спектра тороидальной составляющей отлична. Тем не менее если сравнивать спектрограммы с электростатических зондов и магнитных зондов, то можно отметить относительную идентичность. Это интересно, так как обычно параметрическую неустойчивость изучали с помощью электростатических зондов. В нашем случае параметрическая неустойчивость проявляется не только в виде электростатических колебаний, но и, скорее, электромагнитных колебаний.

На рис. 4 представлены спектры колебаний в зависимости от СВЧ частоты зондирования, что соответствует фактической зависимости от радиуса плазменного шнура. Данная подборка спектрограмм демонстрирует динамику спектра модуляции отраженной СВЧ волны для разных частот, что соответствует отражению волны от слоя плазмы с разной критической плотностью для соответствующих частот. По этим предварительным данным можно сделать вывод о том, что в краевой области плазменного шнура может сильней проявляться распадная параметрическая неустойчивость. При смещении вглубь плазменного шнура сильнее проявляются моды Бернштейна.

Поток убегающих электронов стимулировался дополнительной ВЧ ионизацией, которая проводилась на переднем фронте импульса магнитного поля [9]. ВЧ генератор мощностью $0.5 \mathrm{~kW}$ на частоте $2.45 \mathrm{GHz}$ использовался для создания плазмы, при напряженности магнитного поля, равной $0.8 \mathrm{kOe}$. Характеристики потока убегающих электронов фиксировались по измерениям тока на периферийных зондах Ленгмюра и по выходу жесткого рентгеновского излучения на заднем фронте импульса магнитного поля (рис. 5). 

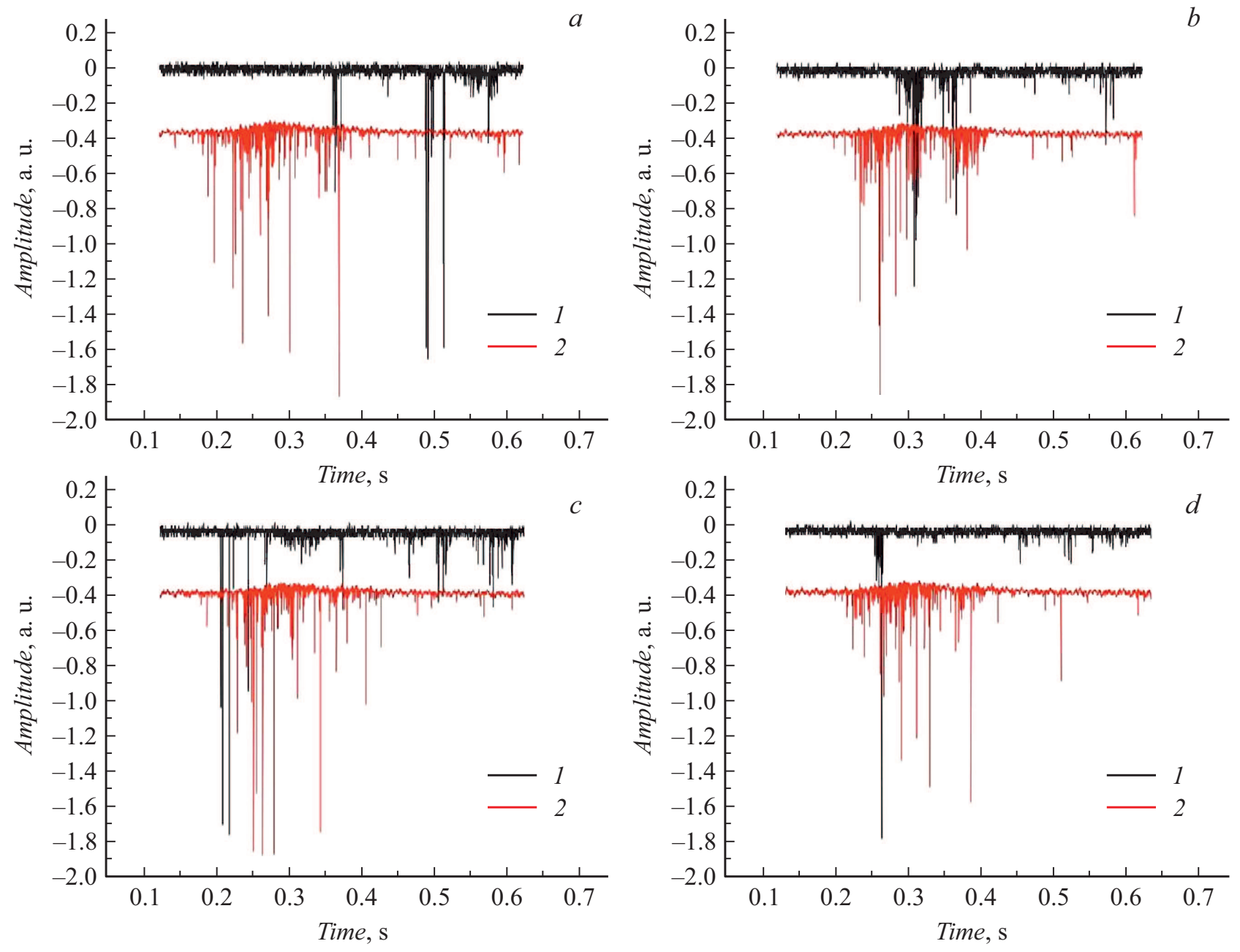

Рис. 8. Сигналы с электростатического зонда (1) для различных гармоник ВЧ частоты накачки: $a-$ первая гармоника, $b-$ вторая гармоника, $c$ - третья гармоника, $d-$ четвертая гармоника; и $c$ датчика рентгеновского излучения $(2)$.

Стимулирование потоков убегающих электронов оказывало влияние не только на условия высокочастотного пробоя газа, но и изменяла порог возбуждения и интенсивность параметрической неустойчивости. Из приведенных осциллограмм видно, что амплитуды как основных гармоник, так и некратных частоте накачки усиливаются с увеличением интенсивности потока убегающих электронов (рис. 6).

При проведении стимулирования происходит трансформация спектра. Некратные гармоники перемещаются в более высокочастотную область. Необходимо отметить, что такое же явление наблюдалось с увеличением вводимой в плазму ВЧ мощности. Данные спектрограммы также указывают на зависимость формы спектра излучения от интенсивности потока убегающих электронов (рис. 7).

В результате наблюдения выхода сгустков частиц, промодулированных частотами, соответствующими частотам мод Бернштейна, был сделан вывод о взаимодействии потока УЭ с ВЧ колебаниями в широком диапазоне частот. Подборка осциллограмм сигналов с пери- ферийных электростатических зондов подтверждает высказанное предположение о взаимодействии потока убегающих электронов с высокочастотным полем антенн генераторов „Каскад-1“ и „Каскад-2“. Поток убегающих электронов претерпевает сильную нелинейную модуляцию в высокочастотном поле или при взаимодействии с возбужденной в плазме волной. Об этом говорит тот факт, что частицы фиксируются датчиками через $1.5-2 \mathrm{~s}$ после окончания ВЧ импульса. Интересно также, что раньше во времени выходят частицы, модулированные более высокими гармониками. Этот факт может свидетельствовать о взаимодействии волна-частица, так как более высокой частоте соответствует большая фазовая скорость волны. В то же время частицы, обладающие большей скоростью, при уменьшении напряженности магнитного поля (задний фронт импульса магнитного поля) быстрее покидают зону удержания и попадают на периферийные зонды. Наблюдаемую картину можно попытаться объяснить образованием слабозатухающих волн (волн Бернштейна-Грина-Крускала) (рис. 8). 


\section{Обсуждение результатов}

В настоящей работе представлены результаты проведенных исследований спектрального состава и динамики электромагнитных флуктуаций с использованием набора высокочастотных электростатических и магнитных зондов, а также результаты, полученные с помощью метода флуктуационной рефлектометрии. Параллельно с этим исследовалась динамика потоков УЭ, которые зарождались на фронтах импульса магнитного поля и существовали в течение всего импульса.

Первые измерения говорят об электромагнитной природе наблюдаемых флуктуаций и наличие их в объеме удержания плазмы.

Экспериментальные исследования указывают на связь возбуждения параметрической неустойчивости и существования потока УЭ в области удержания плазмы торсатрона Ураган-3М.

Необходимо отметить, что возбуждение параметрической неустойчивости отрицательно сказывается на возможности оптимального ввода высокочастотной мощности в плазму. Большая часть вводимой энергии может отражаться, вызывая локальные перенапряжения в месте ввода [10]. Предлагались решения этой проблемы с помощью расширения частотного диапазона вводимой ВЧ энергии или уменьшения локальной напряженности при использовании антенн с развитой поверхностью [11].

Наличие в области удержания потока убегающих электронов также отрицательно сказывается на нагреве и удержании плазмы. При этом могут возникать дополнительные неустойчивости, традиционные для систем пучок-плазма, отбирающие на себя часть вводимой мощности и вызывающие дополнительную диффузию частиц.

\section{Выводы}

1. Изменение частотного спектра исследуемых флуктуаций и их интенсивность связаны с увеличением вводимой в плазму высокочастотной мощности.

2. Наблюдаемые на периферии объема удержания плазмы флуктуации имеют электромагнитную природу.

3. Флуктуации плотности плазмы в частотном диапазоне исследуемой параметрической неустойчивости имеют место в объеме удержания, о чем говорит анализ спектра в частотном диапазоне $(17-24 \mathrm{GHz})$ волн. Это позволяет делать заключение о параметрической природе колебательных процессов, разновидности которых зависят от глубины расположения исследуемого слоя в плазме.

4. Наблюдается связь изменения частотного спектра исследуемых флуктуаций и их интенсивности с интенсивностью потока убегающих электронов, как и в случае с увеличением вводимой в плазму высокочастотной мощности.
5. Спектр модуляций потока убегающих электронов после окончания импульса высокочастотной инжекции соответствует спектру флуктуаций наблюдаемых в плазме и на ее периферии.

\section{Конфоликт интересов}

Авторы заявляют, что у них нет конфликта интересов.

\section{Список литературы}

[1] Olshansky V.V., Stepanov K.N., Tarasov I.K., Tarasov M.I., Sitnikov D.A., Skibenko A.I., Volkov E.D. // Problems of Atomic Sci. Technol. Series: Plasma Phys. 2009. N 1. P. 43-45.

[2] Степанов К.Н., Ольшанский В.В., Тарасов М.И., Ситников Д.А. // Физика плазмы. 2010. Т. 36. № 10. С. 916-925.

[3] Tarasov M.I. Streams of charged particles in plasma and their fluctuations in linear and toroidal magnetic configurations. Kharkov: Thesis, 2011. $140 \mathrm{p}$.

[4] Громов С.Н., Пасечник Л.Л., Семенюк В.Ф. // Письма в ЖЭТФ. 1976. Т. 23. Вып. 3. С. 145-148.

[5] Федорченко В.Д., Киценко А.Б., Серомолот Ю.В., Муратов В.И. // Физика плазмы. 1993. Т. 19. № 1. С. 27-38.

[6] Смолякова О.Б., Токман М.Д. // Физика плазмы. 2000. T. 26. № 9. C. 833-837.

[7] Урузбаев А.О., Вершков В.А., Солдатов С.В., Шелухин Д.А. // Физика плазмы. 2006. Т. 32. № 8. С. 675-698.

[8] Гусаков Е.З., Попов А.Ю., Сурков А.В. ХХХІ Звенигородская конф. по физике плазмы и УТС. 16-20 февраля. 2004.

[9] Тарасов И.К., Тарасов М.И., Ситников Д.А., Пашнев В.К., Лытова М.А. // ЖТФ. 2016. Т. 86. Вып. 1. С. 55-60.

[10] Архипенко В.И., Будников В.Н., Гусаков Е.З., Романчук И.А., Симончик Л.В. // Письма в ЖЭТФ. 1986. Т. 43. Вып. 2. С. 71-73.

[11] Криворучко С.М., Тарасов И.К., Башко В.А. Способ ВЧ-нагрева плазмы и устройство для его осуществления. А.С. № 1158022 от 22 января 1985. 